\title{
Nanobubbles on Electron Transparent Electrodes
}

\author{
Jared J. Lodico ${ }^{1}$, E. R. White ${ }^{1}$, Gavin Carlson ${ }^{1}$, Bradley D. Parks ${ }^{1}$, Alexander Kerelsky ${ }^{1}$, and \\ B. C. Regan ${ }^{1}$ \\ ${ }^{1}$ Department of Physics \& Astronomy and California NanoSystems Institute, University of California, \\ Los Angeles, California 90095 USA
}

The experimental observation of inhomogeneous nanobubble formation is challenging, and consequently the onset and growth of nanobubbles has yet to be fully localized or observed directly. Conventional methods, such as optical microscopy and atomic force microscopy (AFM), have been used to image small bubbles $[1,2]$. However, optical images have limited spatial resolution due to the wavelength of visible light, and AFM is an invasive technique which gives data that can be difficult to interpret. Here we report using an electrical bias applied to graphene electrodes to form nanobubbles in situ for observation with scanning transmission electron microscopy (STEM) .

Through mechanical exfoliation of bulk graphite we isolate single and multi-layered graphene on $\mathrm{Si} / \mathrm{SiO}_{2}$ wafers. The graphene is subsequently transferred to a silicon chip with gold contacts and a 20 $\mathrm{nm}$ thick $\mathrm{SiN}_{\mathrm{x}}$ electron transparent window [3]. To create the fluid cell, a small droplet of liquid is placed between the chip and a second silicon chip without electrodes, which is maneuvered such that the $\mathrm{SiN}_{\mathrm{x}}$ windows are aligned [4]. Applying epoxy around the edges of the cell creates a vacuum-tight seal, which keeps the graphene wet and the electron-transparent windows aligned when the cell is placed in the microscope's high vacuum.

The high-quality, mechanically-exfoliated graphene is an ideal substrate on which to form nanobubbles via Joule-heating. Figure 1 shows optical images of a small bubble forming as a graphene electrode boils the water inside a liquid (water) cell. The graphene sheet contacts two gold electrodes and gives a circuit resistance of $2.2 \mathrm{k} \Omega$. The bubble forms within $34 \mathrm{~ms}$ (one frame) of the application of a $1.6 \mathrm{~V}$ pulse $(300 \mathrm{~ms}$ ), and begins to shrink immediately afterwards (Figure $1 \mathrm{c}-\mathrm{d}$ ). From the positioning of the bubble, which has its centroid between the gold electrodes and not over one of them, it is evident that the bubble nucleated on the graphene itself. Such a device is thus ideal for STEM imaging of thermal nanobubbles, for if the nucleation site can be identified it can be directly imaged with high resolution.

Previously, platinum wires have been used to Joule-heat water and produce nanobubbles for observation with in situ STEM [4]. While a Pt wire effectively creates individual nanobubbles, it most likely obscures the corresponding nucleation sites in the STEM projection image. Here we bias a liquid $\left(\mathrm{H}_{2} \mathrm{SO}_{4}\right)$ cell containing electron-transparent graphene electrodes and image nanobubbles forming. Since in this case the nanobubbles are generated electrochemically via the formation of hydrogen gas $\left(2 \mathrm{H}^{+}+\right.$ $2 \mathrm{e}^{-} \rightarrow \mathrm{H}^{2}(\mathrm{~g})$, the physics underlying bubble nucleation is quite different. Thus graphene shows promise for characterizing the nucleation sites of bubbles produced both thermally and electrochemically.

Figure 2 shows images taken from a 3 minute liquid cell STEM video of electrochemical nanobubble production on a graphene electrode. The graphene electrode is visible, though only barely in Figure 2 b-e; a yellow line has been drawn on the electrode boundary in Figure 2e as a guide to the eye. Nanobubbles are forming in various locations on the graphene, indicating the existence of multiple 
nucleation sites. Efforts are underway to identify and characterize such nucleation sites by exploiting the low background from these electron-transparent graphene electrodes.

\section{References:}

[1] J. W. G. Tyrrell and P. Attard, Phys. Rev. Lett. 87 (2001), 176104.

[2] P. Attard, M. P. Moody, and J. W. G. Tyrrell: Physica A 314 (2002), 696.

[3] B. C. Regan et al., Microscopy and Microanalysis 19 S2 (2013), p. 420.

[4] Edward R. White et al., Appl. Phys. Express 4 (2011), 055201.

[5] This work was supported by NSF DMR-1206849; STARnet, a Semiconductor Research Corporation program sponsored by MARCO and DARPA; and the Electron Imaging Center for NanoMachines at UCLA.

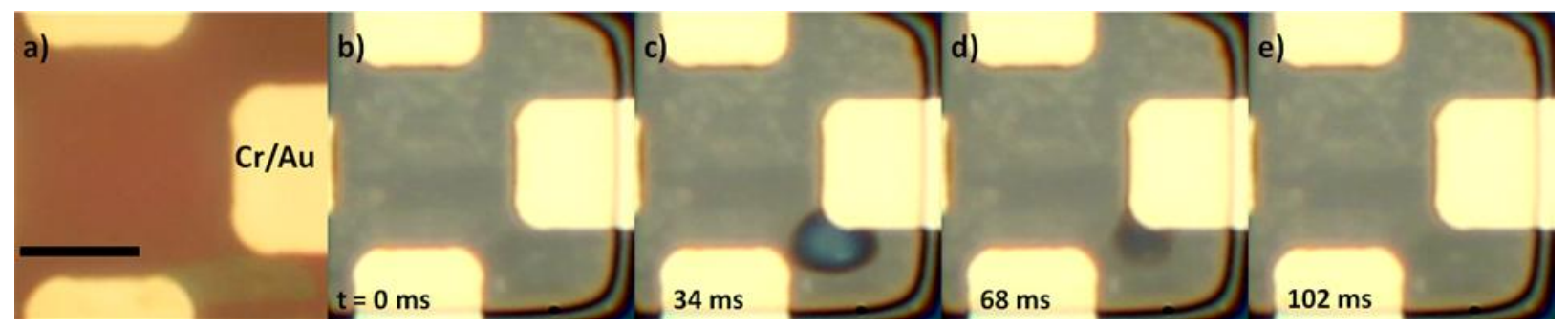

Figure 1. Optical images showing bubbles forming across graphene (approximately 2-5 layers) that is electrically connected to the bottom and right $\mathrm{Cr} / \mathrm{Au}$ electrodes. (a) the graphene is visible over the window before liquid (water) is applied and the cell is sealed with epoxy. The cell shows signs of being wet in images (b) through (e). A $300 \mathrm{~ms}$ pulse of $1.6 \mathrm{~V}$ was applied to create the nanobubble shown in (c). The cell drew $610 \mu \mathrm{A}$ during this pulse. (d) Shows the collapse of the bubble after the pulse has been applied. The scale bar is $10 \mu \mathrm{m}$.

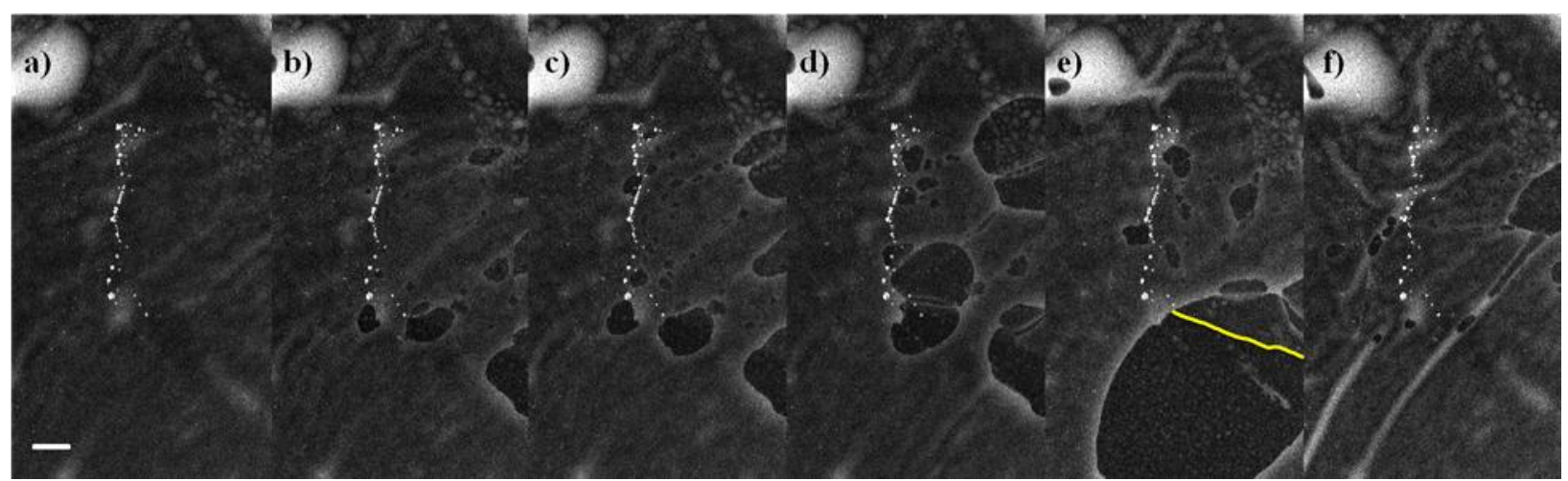

Figure 2. (a)-(f) In situ STEM images $\left(8.2 \times 14.6 \mu \mathrm{m}^{2}\right)$ taken at $5000 \mathrm{x}$ showing nanobubbles. Gas is electrochemically produced in the liquid $\left(\mathrm{H}_{2} \mathrm{SO}_{4}\right)$ by applying a bias between two graphene electrodes. The positive graphene electrode is somewhat visible in images (b)-(e) and is depicted above a yellow outline in (e). The nanobubbles form on the graphene expanding throughout the liquid cell. (f) Wrinkles in the top membrane after bubbles have been made. The scale bar is $1 \mu \mathrm{m}$. 